\title{
Usability Inspection of a Mobile Cancer Telerehabilitation System
}

\author{
Joseph FINKELSTEIN ${ }^{\mathrm{a}}$, Xingyue HUO ${ }^{\mathrm{a}}$, Irena PARVANOVA ${ }^{\mathrm{a}}$, Matthew GALSKY ${ }^{\mathrm{a}}$ \\ ${ }^{a}$ Icahn School of Medicine at Mount Sinai, New York, NY, USA
}

\begin{abstract}
Cancer rehabilitation (CR) has been shown to address physical impairments and progressive disablement experienced by patients with cancer. Access to CR is limited by multiple barriers. Previous studies demonstrated that telemedicine approaches may facilitate access to rehabilitation services however usability and acceptance of cancer telerehabilitation has not been systematically evaluated. This goal of this study was usability inspection of a mobile cancer telerehabilitation system based on cognitive walkthroughs and heuristics evaluations, supplemented by surveys to capture health literacy, patient engagement, and acceptance. The System Usability Scale provided a standardized reference for usability and satisfaction, and the mean result of $83.2 \pm 16.9$ placed this mobile interface in the top 10th percentile. A semi-structured qualitative interview provided actionable feedback, which informed the next iteration of this project. Overall, this mobile telerehabilitation system was considered by cancer patients easy-to-use, satisfying, and engaging with $91 \%$ of participants planning to use it in the future.
\end{abstract}

Keywords. Cancer rehabilitation, telemedicine, usability inspection, mobile health

\section{Introduction}

Cancer rehabilitation has been shown to result in significant improvements in physical, social, psychological and vocational functioning of cancer survivors [1]. Number of cancer survivors is expected to increase due to a growing aging population and an expanding spectrum of effective cancer therapies. It is estimated that the population of cancer survivors will reach 19 million by 2024 [2]. Access to cancer rehabilitation is limited due to multiple existing barriers [3]. Telemedicine approaches can improve access to rehabilitation programs [4] however their acceptance by cancer patients has not been systematically assessed. High acceptance of telerehabilitation by older adults [5] and patients with chronic conditions have been demonstrated in our previous work [6-7]. We designed a telerehabilitation system based on the principles of iterative patientcentered design [8-9]. This project is aimed at assessing usability and acceptance of this system in cancer patients.

\section{Methods}

The study was designed to evaluate the usability and acceptance of the cancer telerehabilitation system by eliciting quantitative and qualitative feedback from cancer patients. Participants were patients with metastatic urogenital cancer receiving outpatient oncology care at the Mount Sinai Health System in New York. Upon sitting down with 
a tablet computer, participants were given a packet of surveys, followed by instructions to complete 3 tasks, then an additional package of evaluations and surveys to record their feedback. The tablet computer included a touchscreen, a stylus, and a keyboard with trackpad, and it was preconfigured to display an icon to $\log$ in to a telerehabilitation system on screen (Figure 1).



Figure 1. User Interface

Surveys began with a socio-demographic form and the Rapid Estimate of Adult Literacy in Medicine (REALM). Participants then performed three tasks as part of the cognitive walkthrough. Afterwards, participants provided feedback on post-task questionnaires, a heuristics evaluation form, and the System Usability Scale (SUS). A semi-structured qualitative exit interview completed the session.

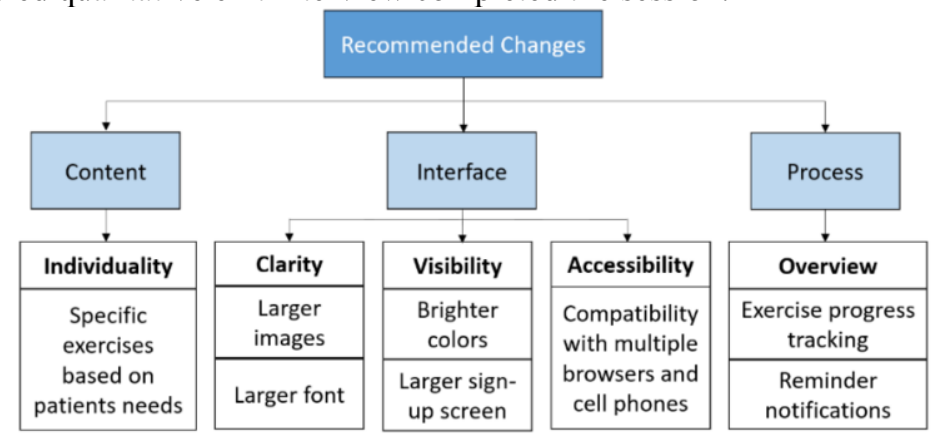

Figure 2. Usability Concept Map of User's Suggestions

The three tasks for the cognitive walkthrough could be completed with or without assistance from the research associate; if a participant needed help, this request was recorded. Task 1 instructed the user how to $\log$ in to the system. Task 2 required users to complete a symptom survey. Task 3 asked users to review exercise plan, and find and perform a particular exercise (sit-to-stand).

After completing the tasks 1 and 2, the participant was asked to grade each task on a scale of 1 (very difficult) to 5 (very easy) using a survey that included the following questions: 1) How difficult or easy was it to review the content and finish the task? 2) How difficult or easy was it to answer the questions/complete the form? 3) How satisfied are you with using this system to complete this task? 4) How would you rate the amount of time it took to complete this task? 5) Is the system visually appealing? 6) Is the system easy to navigate? After completion of the task 3, only questions 3 to 6 were answered. There were also two open-ended questions that ask the participant to share any other feedback and to describe problems they might have encountered. An exit semi-structured qualitative interview followed an interview guide comprising open-ended inquiries on content, interface and process related to the system. 


\section{Results}

Overall, 11 consecutive patients attending genitourinary medical oncology clinic between April and August of 2021 agreed to participate in this study and provide feedback on usability and acceptance of the cancer telerehabilitation system. Average age of the participants was $68.1 \pm 11.2$ years old ranging from 42 to 85 years; 7 participants identified as White $(70 \%), 2$ identified as Black $(20 \%)$, and one did not provide a response for this question; 2 patients identified themselves as Hispanic (18\%); 8 patients reported daily computer use at home (73\%) and 3 patients claimed they never use computer at home (27\%); English proficiency was self-reported as good (18\%) and excellent $(82 \%)$; Participants reported a range of familiarity with cancer rehabilitation with 6 patients $(54.5 \%)$ stating that they never heard about cancer rehabilitation. The average REALM score of $65.5 \pm 0.8$ indicated a 'High School' level of health literacy.

Task self-assessment results are presented in Table 1 as average scores, with a score of 5 indicating highest satisfaction. Overall post-task scores demonstrated high acceptance of the user interface with total scores of $4.7 \pm 0.6 ; 4.6 \pm 0.6 ; 4.7 \pm 0.5$ for tasks $1,2,3$ respectively. The mean satisfaction score for these three tasks was $4.8 \pm 0.4$; $4.6 \pm 0.7 ; 4.9 \pm 0.3$, and the mean "easy to navigate" score was $4.5 \pm 0.7 ; 4.7 \pm 0.5 ; 4.7 \pm 0.5$, respectively. All study subjects (100\%) were able to successfully complete the three test tasks with mean completion time in seconds of $87.5 \pm 101.5 ; 92.5 \pm 93.1 ; 183.1 \pm 502$, respectively. Minor assistance was provided for 3, 1, 3 subjects to complete tasks 1, 2, 3, respectively. The main reason for assistance was difficulties in entering login name and password. Ten out of eleven study subjects $(90.9 \%)$ were certain that they would like to use the cancer telerehabilitation system in the future, one subject was not sure.

The heuristic evaluation demonstrated that the interface functionality is selfexplanatory and can be easily recognized and carried out by the cancer patients. The overall score for heuristics evaluation was $4.5 \pm 0.7$ with the highest score assigned for control and recognition (4.7 \pm 0.5$)$ and the lowest - for aesthetics $(4.2 \pm 1.2)$.

System Usability Scores (SUS) were normalized according to the standard scoring algorithm. Mean SUS was $83.2 \pm 16.9$, which corresponds with an 'above average' usability rating, and places this system usability at greater than the 90th percentile.

The semi-structured qualitative interview captured instructive feedback that described the cancer telerehabilitation platform as easy-to-use, engaging, and supportive for regular exercise. Tailored interactive delivery of multimedia exercise materials was perceived as a major facilitator of daily exercise. The participants stated "I liked the fact that you can either read or watch the video and the video was good. And the video is almost as a partner. You're not alone." and "I like the pictures and videos, even if you don't speak the language very well, you can still do exercises and trust that they can help." Regarding the interface, the patients commented "I am not excessively technology minded, but it was clear to me" and "I didn't really feel that there is necessity for any extra instructions on the exercises. It was very well presented. Once I had my signing in information, I could do it by myself, the whole program is intuitive." The patients believed that the telerehabilitation system "will get you to actually do the exercise." One patient commented: "I think that this will be a great alternative for people like me who are not that much into exercising on a daily and weekly basis." Most of the participants' suggestions were related to improved aesthetics and help options such as: "make the images a little bigger and expand it," "the colors are little washed out," "some guidance may be helpful as I'm not used to touchscreen interface for someone my age, 
"touchscreen with the re-sizing and scrolling requires some learning." A summary of patient suggestions are depicted in Figure 2.

Table 1. Task Self-Assessment

\section{Discussion}

\begin{tabular}{ll}
\hline Task 1: Log in to System & Mean (SD) \\
\hline Login Difficulty & $4.7(0.5)$ \\
Navigation Difficulty & $4.7(0.5)$ \\
Satisfaction & $4.8(0.4)$ \\
Amount of Time & $4.8(0.6)$ \\
Visually Appealing & $4.5(1.0)$ \\
Easy to Navigate & $4.5(0.7)$ \\
Task 2: Complete Symptom Survey & \\
Content Difficulty & $4.6(0.5)$ \\
Questions Difficulty & $4.7(0.5)$ \\
Satisfaction & $4.6(0.7)$ \\
Amount of Time & $4.5(0.8)$ \\
Visually Appealing & $4.5(0.8)$ \\
Easy to Navigate & $4.7(0.5)$ \\
Task 3: Start and Finish Exercise & \\
Satisfaction & $4.9(0.3)$ \\
Amount of Time & $4.5(0.7)$ \\
Visually Appealing & $4.7(0.5)$ \\
Easy to Navigate & $4.7(0.5)$ \\
\hline
\end{tabular}

The mobile cancer telerehabilitation system has been enthusiastically accepted by cancer patients who reported high usability and satisfaction rankings and expressed interest using such a system in the future. The results of usability inspection are largely consistent with our previous work on this platform in older adults [4] and people with chronic neurodegenerative disorders [6].

This is the first study assessing cancer telerehabilitation usability and acceptance in patients with metastatic urogenital cancer. Limitations of this pilot study include a limited sample size, however within this convenience sample patients from broad range of age, computer and health literacy were represented. This limitation will be mitigated in our future studies by broadening the patient base and geographical locations.

\section{Conclusions}

The mobile cancer telerehabilitation platform has demonstrated a high usability rating with excellent levels of user-reported satisfaction. Next steps should include addressing patient suggestions on improving the system functionality and evaluating clinical impact of cancer telerehabilitation in a randomized controlled trial.

\section{References}

[1] Cheville AL, Mustian K, Winters-Stone K, et al. Cancer Rehabilitation: An Overview of Current Need, Delivery Models, and Levels of Care. Phys Med Rehabil Clin N Am. 2017 Feb;28(1):1-17.

[2] American Cancer Society: Cancer treatment \& survivorship: facts \& figures 2014-2015, Atlanta, 2015. 
[3] Alfano CM, Cheville AL, Mustian K. Developing High-Quality Cancer Rehabilitation Programs: A Timely Need. Am Soc Clin Oncol Educ Book. 2016;35:241-9.

[4] Jeong IC, Liu J, Finkelstein J. Association between System Usage Pattern and Impact of WebBased Telerehabilitation in Patients with Multiple Sclerosis. Stud Health Technol Inform. 2020;272:346-349.

[5] Finkelstein J, Liu J. Usability of Telerehabilitation System Supporting Multipronged Exercise in Patients with Multiple Sclerosis. Stud Health Technol Inform. 2018;251:281-284.

[6] Jeong IC, Liu J, Finkelstein J. Factors Affecting Adherence with Telerehabilitation in Patients with Multiple Sclerosis. Stud Health Technol Inform. 2019;257:189-193.

[7] Finkelstein J, Liu J. Designing Telerehabilitation System for Multipronged Exercise in Patients with Multiple Sclerosis. Stud Health Technol Inform. 2018;254:16-23.

[8] Bedra M, Finkelstein J. Feasibility of post-acute hip fracture telerehabilitation in older adults. Stud Health Technol Inform. 2015;210:469-73.

[9] Finkelstein J, Jeong IC, Doerstling M, et al. Usability of Remote Assessment of Exercise Capacity for Pulmonary Telerehabilitation Program. Stud Health Technol Inform. 2020;275:72-76. 